\title{
Editorial: 5G Wireless Communication Systems and Advanced Image Processing
}

\author{
Song Guo ${ }^{1} \cdot$ Baoliu Ye ${ }^{2}$ \\ Published online: 4 February 2021 \\ (C) The Author(s), under exclusive licence to Springer Science+Business Media, LLC part of Springer Nature 2021
}

\section{Editorial:}

As widely acknowledged, a future wireless network will not only support a fast growing overall mobile data volume and a significantly increased number of connected devices, but also adapt to dynamically fluctuating traffic demands and a great deal of new requirements of future service portfolios. To satisfy the above trends, mobile stakeholders are already preparing the technology roadmap for " $5 \mathrm{G}$ ", which promises to meet all sorts of diverse and extreme requirements.

The aim of this special issue is to bring together researchers and developers as well as regulators and policy makers to present their latest views on 5G: new networking, new wireless communications, resource control and management, and new emerging applications, and more.

The first group on new networking includes the following two papers. In the article "A Network Calculus Based Delay and Backlog Analysis for Cloud Radio Access Networks", the authors studied delay and backlog upper bounds in C-RAN. They classified applications into three priorities in terms of different required delay tolerance, to reflect the real scenario in $5 \mathrm{G}$ cellular mobile communication networks. By applying network calculus, this paper derived a closed form expression for delay and backlog upper bounds. Ultra-Reliable Low Latency Communication (URLLC) is one of the classic scenarios in 5G. In the second article "Performance Evaluation of URLLC in 5G Based on Stochastic Network Calculus", the authors build a tandem model to describe the communication

Song Guo

song.guo@polyu.edu.hk

Baoliu Ye

yebl@hhu.edu.cn

1 Hong Kong Polytechnic University, 11 Yuk Choi Rd, Hung Hom, Hongkong

2 Division of Information, Hohai University, 1 Xi Kang Lu, Gulou, Nanjing, Jiangsu, China in the $5 \mathrm{G}$ network and use the stochastic network calculus and take into account the features of URLLC network to analyze the delay. The parameters which influence the delay are researched. The results derived from the evaluation reveals how to optimize the parameters to reduce the delay, which would provide valuable guidelines for the design of URLLC architecture.

The second group on new wireless communications includes the following three papers. In the article "UserCentered Interference Coordination in the Ultra-Dense Network: a Cluster and Priority Perspective", the authors studied the serious multi-cell interference problem in the ultradense network, due to the serious interferences caused by the high node density and random distributions of nodes in small cells. To solve this problem, a novel scheme is proposed with clustered priority-based user centered interference coordination. Herein, the random interference coordination is transformed into prioritized process in the ultra-dense network. Afterwards, the whole interfering nodes are divided into three cluster sets. The first cluster set with coordinated multipoint transmission technology is used to provide useful signal. The second cluster set works with zero-forcing process. The studies have shown that the total interference can be decreased significantly so that the system performance can be improved obviously. The next article titled "Optimization of FBMC Waveform by Designing NPR Prototype Filter with Improved Stopband Suppression" proposed a near perfect reconstruction (NPR) prototype filter design for the promising waveform FBMC. In this design, the original non-convex problem is circumvent based on coefficient autocorrelation. Then the Nyquist condition can be easily relaxed for better stopband suppression, which results better BER performance in comparison with conventional prototype filter (PHYDYAS and IOTA). The improved stopband suppression makes FBMC a good candidate for asynchronous multiple access and fragmented spectrum communications in the future wireless communication. The last article titled "Robust SpectrumEnergy Efficiency for Green Cognitive Communications", proposed a novel multichannel network model in which 
cognitive users are incorporated with the capacity of opportunistically harvesting radio energy. In this framework structure, cognitive radio users adopt the dual cooperative spectrum sensing scheme to periodically sense the status of primary users whether exist or not in multi-bands and harvest the radio frequency energy from primary users when they transmit data, or else, cognitive radio users can occupancy this frequency. Then, formulate spectrum and energy efficiency function with respect to transmission power, dynamic cooperative sensing time, and channel state. Using spectrum sharing and convex optimization techniques, robust optimal power and channel allocations are proposed.

The third group on resource control and management includes the following two papers. The article titled "EnergyEfficient Power Allocation Scheme for Uplink Distributed Antenna System with D2D Communication" presented the optimization problem for energy efficiency (EE) maximization by considering both the total power constraint and the rate constraints and proposed an energy-efficient power allocation (PA) scheme based on the bisection method. The proposed optimal PA scheme can achieve better EE performance than the conventional equal PA scheme, and the same EE as the PA scheme based on two-dimensional search method but with lower complexity. In the next article with the title "ClusterBased Joint Resource Allocation with Successive Interference Cancellation for Ultra-Dense Networks", the authors formulated an optimization problem based on joint clustering, subchannel allocation and successive cancellation among users by considering all types of interference and quality of service (QoS) requirements of users. To solve this optimization problem, an interference graph based clustering algorithm was first proposed to distribute femto base stations (FBSs) and femto user equipments (FUEs) into disjoint clusters and groups synchronously. Then, to minimize the cross-tier interference, a suboptimal heuristic algorithm was provided to allot the subchannel to each FBSs cluster. Moreover, detecting and demodulating the largest received power of FUEs successively, an ordered successive interference cancellation (OSIC) detection algorithm was developed to reduce the interference among users in the same FUE group. Numerical results validate the average capacity and spectral efficiency of the proposed scheme have been improved in different network scenarios.

The last group is on new emerging applications including the following three papers. The article with the title "Cooperation of Mobile Devices for Fast Inference of Deep
Learning Applications" studied the collaborative inference problem among mobile devices, the authors let mobile devices collaboratively share the computation workloads instead of sending deep learning inference tasks to cloud. This work is based on an important observation that batching inference tasks on GPUs can reduce the average inference time. In the collaborative mode, any mobile device might become an aggregator node that receives the tasks from its neighbors via direct link technology and execute the tasks with sharing its computation resources. The next article titled "An Effective Algorithm for Single Image Fog Removal" proposed an effective algorithm for single image fog removal based on degradation model and group-based sparse representation (GSR). The proposed degradation model is constructed based on a classical physical model, i.e., dichromatic atmospheric scattering model. Then, the new degradation model is integrated into the group-based sparse representation framework. Finally, the single image defogging problem is regarded as an image restoration problem, which can be well optimized by GSR. The method is compared with several well-known algorithms from the literature using qualitative and quantitative evaluations, and results indicate considerable improvement over existing algorithms. The last article titled "An Object Recognition Approach for Synthetic Aperture Radar Images" proposed a synthetic aperture radar (SAR) target recognition algorithm based on the enhanced kernel sparse representation. It combines the advantages of monogenic features and the kernel discriminant analysis to form a new discriminative feature mapping approach, which exploits the augmented pseudo-transformation matrix to reduce the data dimensionality in the kernel feature space effectively. Then features are fed into enhanced kernel sparse representation-based classifier to reach the inference of SAR target with high recognition accuracy.

In conclusion, the papers presented in this special issue demonstrate the breadth and diversity of research in the field of $5 \mathrm{G}$ Wireless Communication Systems and Advanced Image Processing. We wish to thank the authors and the reviewers for their hard work in helping us assemble this special issue. We would also like to express our sincere gratitude to the Editor-in-Chief, Professor Imrich Chlamtac, for providing this opportunity and tremendous guidance throughout the process.

Publisher's note Springer Nature remains neutral with regard to jurisdictional claims in published maps and institutional affiliations. 


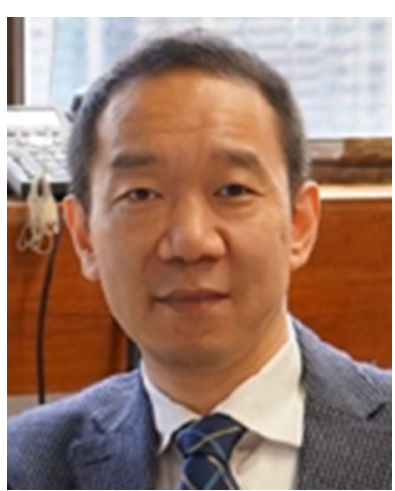

Song Guo is a Full Professor in the Department of Computing at The Hong Kong Polytechnic University. His research interests are mainly in the areas of big data, edge AI, mobile computing, and distributed systems. He coauthored 4 books, co-edited 7 books, and published over 500 papers in major journals and conferences. He is the recipient of 12 Best Paper Awards from various IEEE/ACM conferences, journals, and technical committees. His work was also recognized by the 2016 Annual Best of Computing: Notable Books and Articles in Computing in ACM Computing Reviews. Prof. Guo's research has been sponsored by RGC, NSFC, MOST, JSPS, industry, etc. Prof. Guo is the Editor-in-Chief of IEEE Open Journal of the Computer Society and the Chair of IEEE Communications Society (ComSoc) Space and Satellite Communications Technical Committee. He was an IEEE ComSoc Distinguished Lecturer, a member of IEEE ComSoc Board of Governors, and a member of Fellow Evaluation Committee of IEEE Computer Society. Prof. Guo has been named on editorial board of a number of prestigious international journals like IEEE Transactions on Parallel and Distributed Systems, IEEE Transactions on Cloud Computing, IEEE Internet of Things Journal, etc. He has also served as chairs of organizing and technical committees of many international conferences. Prof. Guo is an IEEE Fellow (Computer Society), a Highly Cited Researcher (Web of Science), and an ACM Distinguished Member.

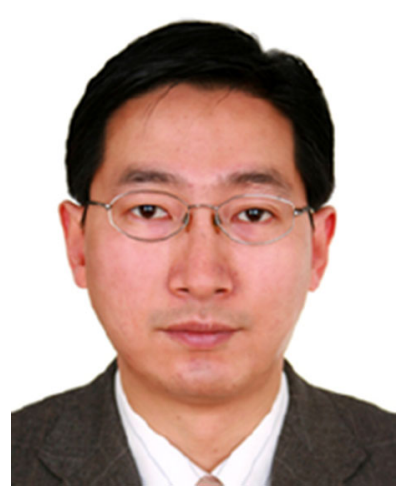

Baoliu Ye received the Ph.D. degree in computer science from Nanjing University, China, in 2004 . He was a Visiting Researcher with The University of Aizu, Japan, from 2005 to 2006. He is now serving as the director of the Division of Information, Hohai University, since 2018. He is currently a Full Professor with the Department of Computer Science and Technology, Nanjing University. He has published more than 100 articles in major conferences and journals. His current research interests mainly include distributed systems, edge computing, and wireless networks. He is the Regent of CCF and the Secretary-General of the CCF Technical Committee of Distributed Computing and Systems. 